Nota / Note

\title{
NÚMERO DE ÍNSTARES LARVAIS DE Tuta absoluta (MEYRICK) EM GENÓTIPOS DE TOMATEIRO
}

\author{
Teresinha Augusta Giustolin*; José Djair Vendramim; José Roberto Postali Parra \\ Depto. de Entomologia, Fitopatologia e Zoologia Agrícola, USP/ESALQ, C.P. 9 - CEP: 13418-900 - Piracicaba, SP. \\ *Autor correspondente <tagiusto@carpa.ciagri.usp.br>
}

RESUMO: A traça Tuta absoluta (Meyrick) é uma das principais pragas do tomateiro no Brasil e tem sido controlada quase que exclusivamente com inseticidas, o que é indesejável tanto por motivos econômicos quanto ambientais. Esses problemas podem ser minimizados com métodos alternativos de controle, tal como a resistência varietal que é uma alternativa interessante, mas que ainda precisa ser mais estudada. Visando obter mais informações sobre a relação inseto-planta, estudou-se o número de instares da traça em dois genótipos de tomateiro Lycopersicon hirsutum f. glabratum (PI 134417 - resistente) e L. esculentum ('Santa Clara' - suscetível). O experimento foi desenvolvido em câmara climatizada em duas temperaturas $(26 \pm 0,5$ e $30 \pm 0,5^{\circ} \mathrm{C}$ ). As lagartas recém-eclodidas foram alimentadas com folhas dos dois genótipos. Diariamente foram retiradas 15 lagartas de cada tratamento para medições da largura da cápsula cefálica. O desenvolvimento do inseto seguiu a regra de Dyar e, em qualquer das situações testadas, apresentou quatro ínstares larvais.

Palavras-chave: Gelechiidae, traça-do-tomateiro, resistência de plantas, ínstares larvais

\section{NUMBER OF LARVAL INSTARS OF Tuta absoluta (MEYRICK) IN TOMATO GENOTYPES}

\begin{abstract}
The South American tomato pinworm, Tuta absoluta (Meyrick), is one of the most important tomato pests in Brazil and it has been controlled mainly by insecticides, which may be undesirable because of economical and environmental concerns. These problems can be minimized with alternative control tactics, such as the use of the resistant varieties. However, there is a lack of studies on alternative tactics to control $T$. absoluta. To obtain information on the insect-plant interactions, the number of instars of $T$. absoluta on two tomato genotypes: Lycopersicon hirsutum f. glabratum (PI 134417 - resistant) and L. esculentum ('Santa Clara' - susceptible) were determined. The experiment was carried out in climatic chambers, using two temperatures the $26 \pm 0,5$ and $30 \pm 0,5^{\circ} \mathrm{C}$. Neonate larvae were fed with leaves of each genotype. Fifteen larvae of each treatment were removed daily to measure the width of the cephalic capsule. The development of the insect followed the Dyar's rule and four larval instars were found in all treatments.
\end{abstract}

Key words: Gelechiidae, south American tomato pinworm, plant resistance, larval instar

\section{INTRODUÇÃO}

A traça-do-tomateiro Tuta absoluta (Meyrick) representa um sério problema à tomaticultura, não somente pela intensidade de ataque mas também por sua ocorrência durante todo o ciclo da cultura. O controle desta praga é feito quase que exclusivamente através do uso de inseticidas, ocasionando gastos elevados (Souza \& Reis, 1992). Outros métodos alternativos ao controle químico podem ser usados, tal como a resistência de plantas a insetos, que é compatível com outras estratégias utilizadas no manejo integrado.

Dentre os genótipos de tomateiro resistentes a pragas, tem se destacado a linhagem PI 134417 (Lycopersicon hirsutum f. glabratum) que também apresenta resistência a $T$. absoluta (Lourenção et al., 1985; Giustolin \& Vendramim, 1994; Maluf et al., 1997; Gontijo-Lebory et al., 1999; Leite et al., 1999), podendo, em razão disso, ser utilizado como fonte de resistência a essa praga. Nos estudos de resistência varietal, é interessante se obter a maior quantidade possível de informações sobre o inseto para melhor caracterizar os mecanismos de resistência envolvidos. Um dos aspectos muito pouco estudados em relação à traça-do-tomateiro é o que se refere ao número de ínstares, já que esse número pode ser influenciado por diversos fatores como hereditariedade, forma de criação, temperatura, nutrição, sexo etc. (Parra \& Haddad, 1989).

Esse trabalho teve como objetivo determinar o efeito de dois genótipos de tomateiro (um resistente à praga e um suscetível) e de duas temperaturas sobre o número de ínstares do inseto.

\section{MATERIAL E MÉTODOS}

O trabalho foi desenvolvido em câmaras climatizadas reguladas a $26 \pm 0,5$ e $30 \pm 0,5^{\circ} \mathrm{C}$, UR de $80 \pm 10 \%$ e fotofase de 14 horas com lagartas de $T$. 
absoluta alimentadas com folhas dos genótipos de tomateiro L. hirsutum f. glabratum (PI 134417 - resistente) e L. esculentum (cv. Santa Clara - suscetível).

Os genótipos foram cultivados em casa de vegetação em vasos com solo esterilizado e adubado, sendo as plantas utilizadas quando estavam na fase de desenvolvimento vegetativo com 4-5 folhas completamente desenvolvidas. Visando à obtenção de insetos em diferentes estágios de desenvolvimento, lagartas foram alimentadas em folhas, completamente desenvolvidas, dos dois genótipos de tomateiro. Para isso, as folhas das plantas foram colocadas em placas de Petri plásticas $(6,5 \times 2,5 \mathrm{~cm})$ e infestadas com 20 lagartas recém-eclodidas por placas, obtidas de criação de laboratório e colocadas em cada uma das temperaturas. Para cada um dos genótipos, na temperatura de $30^{\circ} \mathrm{C}, 12$ placas foram infestadas com lagartas e na de $26^{\circ} \mathrm{C} 16$ placas, sendo utilizada um total de 1120 lagartas. Folhas novas dos genótipos foram fornecidas às lagartas a cada 2 ou 3 dias.

Diariamente, 15 lagartas de cada um dos tratamentos eram retiradas do interior das minas com o auxílio de um estilete de ponta fina e mortas em água quente e fixadas em álcool $70 \%$, sendo esse procedimento mantido até a pupação. Posteriormente, foram medidas as larguras das cápsulas cefálicas com o auxílio de uma ocular graduada, acoplada a um microscópio estereoscópico. O delineamento experimental, inteiramente casualizado, constou de quatro tratamentos: 'Santa Clara' (a 26 e a $30^{\circ} \mathrm{C}$ ) e PI 134417 (a 26 e a $30^{\circ} \mathrm{C}$ ).

A razão de crescimento $(K)$ e o coeficiente de determinação $\left(R^{2}\right)$ foram determinados através do software MOBAE (Haddad et al., 1995). Foi calculado ainda, o tempo médio necessário para as lagartas atingirem cada ínstar, a partir da eclosão. Para determinação do número de ínstares, considerou-se a curva multimodal, a sobreposição dos intervalos de confiança $(P<0,05)$ das larguras de cápsulas cefálicas entre ínstares sucessivos, o maior $\mathrm{R}^{2}$ e o valor de $\mathrm{K}$.

\section{RESULTADOS E DISCUSSÃO}

A partir das curvas de distribuição multimodal de larguras das cápsulas cefálicas de lagartas de $T$. absoluta (Figuras 1A, 1B, 1C e 1D), observa-se a existência de quatro picos de freqüência. Observa-se ainda, um aumento na largura das cápsulas, com o avanço dos ínstares larvais tanto para lagartas criadas na cultivar Santa Clara como para as alimentadas na linhagem PI 134417. Para as duas temperaturas $\left(26\right.$ e $\left.30^{\circ} \mathrm{C}\right)$, é rejeitada a hipótese da existência de cinco ínstares. Os resultados dos cálculos de sobreposição dos intervalos de confiança das larguras de cápsulas cefálicas não demonstram a ocorrência desse fato em relação à hipótese de quatro ínstares (Tabelas 1 e 2). A elevação na temperatura que acarreta aumento no número de

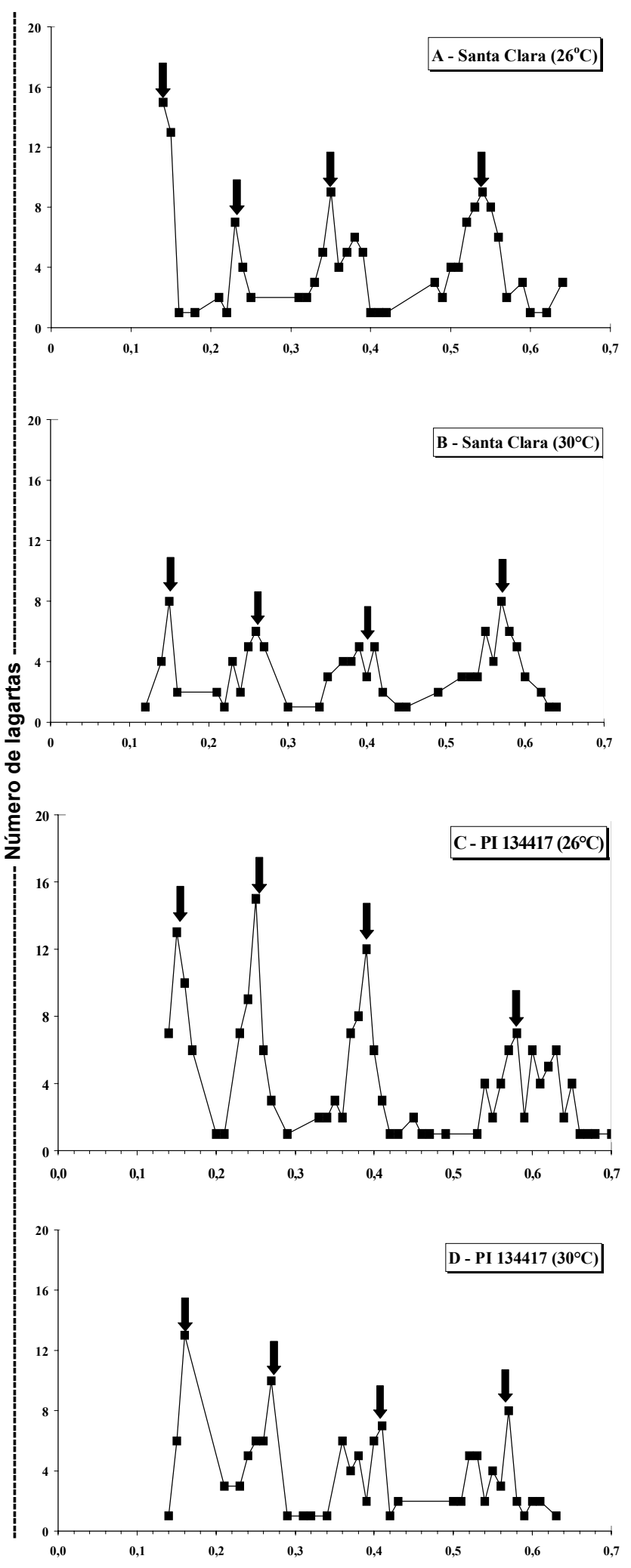

Largura da cápsula cefálica $(\mathrm{mm})$

Figura 1 - Curva de distribuição multimodal da largura das cápsulas cefálicas de lagartas de Tuta absoluta alimentadas em folhas de: A) Santa Clara $\left(26^{\circ} \mathrm{C}\right)$; B) Santa Clara $\left(30^{\circ} \mathrm{C}\right)$; C) PI $134417\left(26^{\circ} \mathrm{C}\right)$; D) PI $134417\left(30^{\circ} \mathrm{C}\right)$. Fotofase: $14 \mathrm{~h}$ e UR: $80 \pm 10 \%$. 
Tabela 1 - Largura das cápsulas cefálicas de lagartas de Tuta absoluta criadas em folhas do cv. Santa Clara, a 26 e $30^{\circ} \mathrm{C}$.

\begin{tabular}{|c|c|c|c|c|c|c|}
\hline \multirow{3}{*}{ Ínstar } & \multicolumn{6}{|c|}{ Largura } \\
\hline & \multicolumn{3}{|c|}{$26^{\circ} \mathrm{C}$} & \multicolumn{3}{|c|}{$30^{\circ} \mathrm{C}$} \\
\hline & $\mathrm{N}^{1}$ & Média & $I^{1}{ }^{1}(P<0,05)$ & $\mathrm{N}^{1}$ & Média & $I^{2}(P<0,05)$ \\
\hline & 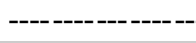 & --.-- & 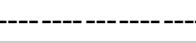 & 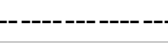 & - & 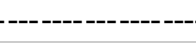 \\
\hline I & $0,14-0,18$ & 0,15 & $0,13-0,17$ & $0,12-0,22$ & 0,16 & $0,13-0,19$ \\
\hline$\|$ & $0,21-0,25$ & 0,24 & $0,22-0,24$ & $0,23-0,34$ & 0,26 & $0,23-0,29$ \\
\hline III & $0,31-0,42$ & 0,36 & $0,34-0,38$ & $0,35-0,45$ & 0,39 & $0,37-0,41$ \\
\hline N & $0,48-0,64$ & 0,54 & $0,51-0,57$ & $0,49-0,64$ & 0,57 & $0,55-0,59$ \\
\hline $\mathrm{R}^{2}$ & 1,00 & - & - & 1,00 & - & - \\
\hline $\mathrm{K}$ & 1,50 & - & - & 1,48 & - & - \\
\hline
\end{tabular}

${ }^{1} I V$ - Intervalo de variação; ${ }^{2} \mathrm{IC}$ - Intervalo de confiança.

ínstares de Tenebrio molitor e reduz esse número para Alabama argillacea (Parra \& Haddad, 1989), não afetou o número de ínstares da traça, que foi sempre quatro. Além da temperatura, também a alimentação dos insetos pode afetar essa variável (Parra \& Haddad, 1989). Um maior número de ínstares apresentado por um inseto está correlacionado à inadequação nutricional (Slansky \& Rodriguez,1987) .

A partir da regra de Dyar (Haddad et al., 1995), os resultados da hipótese da existência de quatro ínstares demonstraram que o coeficiente de determinação $\left(R^{2}\right)$ foi de $100 \%$, para todos os tratamentos (Tabela 1 e 2). Esses resultados comprovam que os intervalos de medida de cápsula formulados apresentaram alta qualidade no ajuste.

$E m$ relação às razões de crescimento $(K)$ (Haddad et al., 1995), para a hipótese de quatro ínstares, os valores variaram de 1,45 a 1,55 , para todos os tratamentos (Tabelas 1 e 2). Portanto, as distribuições dos valores de larguras de cápsulas cefálicas de $T$. absoluta seguem a regra de Dyar. Os valores de intervalo de variação das larguras de cápsula cefálica foram bastante próximos para os quatro tratamentos (Tabelas 1 e 2).

Em tomateiro comercial, Vargas (1970) obteve quatro ínstares larvais para a traça, com valores de largura de cápsula cefálica que variaram de $0,15 \mathrm{~mm}$ para $\circ 1^{\circ}$ ínstar a $0,834 \mathrm{~mm}$ para $04^{\circ}$ ínstar. Razuri \& Vargas (1975) fizeram as medidas das cápsulas cefálicas de duas gerações de laboratório, observando constância em ambos os registros, com valores que variaram de $0,16 \mathrm{~mm}$ para o $1^{\circ}$ ínstar a $0,55 \mathrm{~mm}$ para o $4^{\circ}$ ínstar. A discrepância observada entre os resultados de Razuri \& Vargas (1975) e o de Vargas (1970) quanto à medida do diâmetro da cápsula do $4^{\circ}$ ínstar $(0,55$ e $0,834 \mathrm{~mm}$, respectivamente) é sugerida pelos autores do primeiro trabalho como erro no trabalho de Vargas (1970), no qual foi registrado para o $3^{\circ}$ ínstar valor de $0,40 \mathrm{~mm}$. Nos demais trabalhos, os autores obtiveram valores que giravam em torno de 0,50 a $0,60 \mathrm{~mm}$ para o $4^{\circ}$ ínstar. Quiroz (1976), embora não tenha utilizado a distribuição de freqüência multimodal, obteve, por meio da medida das cápsulas cefálicas, quatro ínstares larvais para a traça. Assim, tanto em mensurações do comprimento e diâmetro do corpo (Coelho \& França, 1987) como em medições de largura da cápsula cefálica das lagartas (Haji et al., 1988; Ferreira \& Anjos, 1997; Minsfeldt, 1998), foram observados quatro ínstares larvais para $T$. absoluta.

A resistência do tomateiro à traça tem sido observada em materiais selvagens, comerciais e em cruzamentos destes materiais, sendo detectado em maior nível nos materiais não comerciais. A linhagem PI 134417 do tomateiro selvagem L. hirsutum f. glabratum tem sido citada como resistente a diversas espécies de insetos pragas (Juvik et al., 1982; Kennedy \& Sorenson, 1985; Farrar Jr. \& Kennedy, 1987, 1988, 1991), incluindo a traça-do-tomateiro (Lourenção et al., 1985; Giustolin \& Vendramim, 1994; Maluf et al., 1997; Gontijo-Lebory et al., 1999). Essa linhagem causa alongamento das fases larval e pupal, redução da viabilidade larval, menor peso de pupas e menor fecundidade das fêmeas de $T$. absoluta em relação ao cultivar Santa Cruz Kada (Giustolin \& Vendramim, 1994). Esse alongamento da fase larval poderia acarretar variação no número de ínstares, como foi observado por Reis Filho et al. (1986), que alimentando lagartas de Erinnyis ello ello (L.) com folhas de diversos clones de seringueira, constataram maior número de ínstares em clones resistentes que alongaram o desenvolvimento do referido inseto. No caso da traça, entretanto, o alongamento da fase larval não foi o suficiente para causar mudança no número de ínstares. Apesar de não ter ocorrido aumento nesse número, a alimentação na $\mathrm{PI} 134417$ provocou maior suscetibilidade do inseto aos entomopatógenos Bacillus thuringiensis e Beauveria bassiana (Giustolin, 1996).

No cv. Santa Clara, os tempos médios necessários para o inseto atingir o $2^{\circ}, 3^{\circ}$ e $4^{\circ}$ ínstares foram menores do que na linhagem PI 134417, indicando que esse material é menos adequado para o desenvolvimento do inseto, confirmando pesquisas anteriores (Giustolin \& Vendramim, 1994; Giustolin, 1996) (Figura 2). $\mathrm{O}$ aumento da temperatura de 26 para $30^{\circ} \mathrm{C}$ 
Tabela 2 - Largura das cápsulas cefálicas de lagartas de Tuta absoluta criadas em folhas da linhagem PI 134417 a 26 e $30^{\circ} \mathrm{C}$.

\begin{tabular}{|c|c|c|c|c|c|c|}
\hline \multirow{3}{*}{ Ínstar } & \multicolumn{6}{|c|}{ Largura } \\
\hline & \multicolumn{3}{|c|}{$26^{\circ} \mathrm{C}$} & \multicolumn{3}{|c|}{$30^{\circ} \mathrm{C}$} \\
\hline & $\mathrm{N}^{1}$ & Média & $\mathrm{IC}^{1}(P<0,05)$ & $\mathrm{N}^{1}$ & Média & $\mathrm{IC}^{2}(P<0,05)$ \\
\hline & \multicolumn{6}{|c|}{ 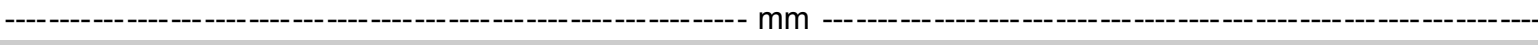 } \\
\hline I & $0,14-0,20$ & 0,16 & $0,14-0,18$ & $0,14-0,21$ & 0,16 & $0,13-0,19$ \\
\hline$\|$ & $0,21-0,29$ & 0,25 & $0,23-0,27$ & $0,23-0,31$ & 0,26 & $0,24-0,28$ \\
\hline III & $0,33-0,47$ & 0,39 & $0,37-0,41$ & $0,32-0,43$ & 0,39 & $0,37-0,41$ \\
\hline $\mathrm{N}$ & $0,49-0,71$ & 0,60 & $0,57-0,63$ & $0,50-0,63$ & 0,55 & $0,53-0,57$ \\
\hline $\mathrm{R}^{2}$ & 1,00 & - & - & 1,00 & - & - \\
\hline $\mathrm{K}$ & 1,55 & - & - & 1,45 & - & - \\
\hline
\end{tabular}

${ }^{1} \mathrm{IV}$ - Intervalo de variação; ${ }^{2} \mathrm{IC}$ - Intervalo de confiança

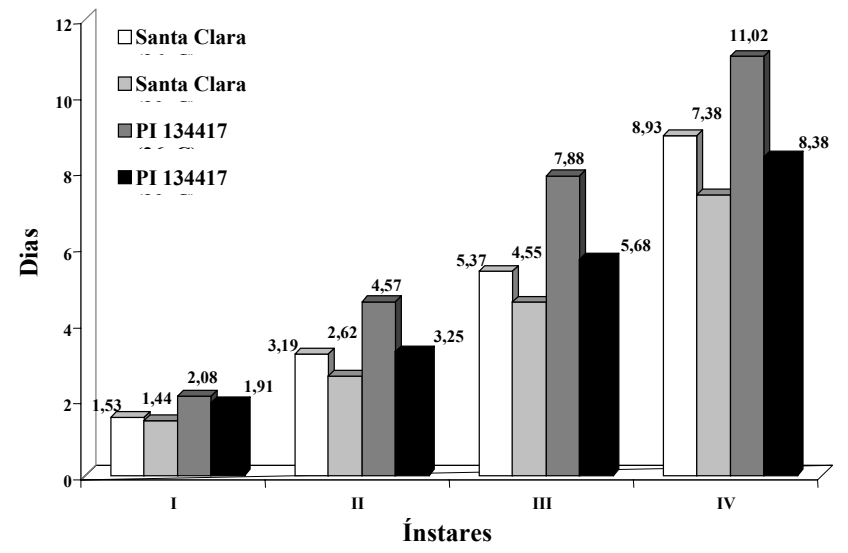

Figura 2 - Tempo médio de cada ínstar larval de Tuta absoluta alimentada com folhas do cv. Santa Clara e da linhagem PI 134417, a 26 e $30^{\circ} \mathrm{C}$, fotofase: 14 h e UR: $80 \pm 10 \%$.

reduziu os referidos tempos. Apesar de esses tratamentos terem afetado o desenvolvimento de $T$. absoluta, esses efeitos não foram tão acentuados a ponto de influenciar o número de ínstares que foi quatro em todas as situações testadas.

\section{REFERÊNCIAS BIBLIOGRÁFICAS}

COELHO, M.C.F.; FRANÇA, F.H. Biologia e quetotaxia da larva e descrição da pupa e adulto da traça-do-tomateiro. Pesquisa Agropecuária Brasileira, v.22, p.129-135, 1987.

FARRAR JUNIOR, R.R.; KENNEDY, G.G. Growth, food consumption and mortality of Heliothis zea larvae on foliage of the wild tomato Lycopersicon hirsutum f. glabratum and the cultivated tomato, L. esculentum. Entomologia Experimentalis et Applicata, v.44, p.213-219, 1987.

FARRAR JUNIOR, R.R.; KENNEDY, G.G. 2-undecanone, a pupal mortality factor in Heliothis zea: sensitive larval stage and in plant activity in Lycopersicon hirsutum f. glabratum. Entomologia Experimentalis et Applicata, v.47, p.205-210, 1988.

FARRAR JUNIOR, R.R.; KENNEDY, G.G. Relationship of leaf lamellar-based resistance to Leptinotarsa decemlineata and Heliothis zea in a wild tomato, Lycopersicon hirsutum f. glabratum, PI 134417. Entomologia Experimentalis et Applicata, v.58, p.61-67, 1991.

FERREIRA, J.A.M.; ANJOS, N. Caracterização dos ínstares larvais de Tuta absoluta (Meyrick) (Lepidoptera: Gelechiidae) In: CONGRESSO BRASILEIRO DE ENTOMOLOGIA, 16., Salvador, 1997. Resumos. Salvador: SEB, 1997. p.64.

GIUSTOLIN, T.A. Efeito de dois genótipos de Lycopersicon spp. associados aos entomopatógenos Bacillus thuringiensis var. kurstaki e Beauveria bassiana no desenvolvimento de Tuta absoluta (Meyrick, 1917) (Lep., Gelechiidae). Piracicaba, 1996. 99p. Tese (Doutorado) - Escola Superior de Agricultura "Luiz de Queiroz", Universidade de São Paulo.
GIUSTOLIN, T.A.; VENDRAMIM, J.D. Efeito de duas espécies de tomateiro na biologia de Scrobipalpuloides absoluta (Meyrick). Anais da Sociedade Entomológica do Brasil, v.23, p.511-517, 1994.

GONTIJO-LABORY, C.R.; SANTA-CECÍLIA, L.V.C.; MALUF, W.R.; CARDOSO, M. das G.; BEARZOTTI, E.; SOUZA, J.C. de. Seleção indireta para teor de 2-tridecanona em tomateiros segregantes e sua relação com a resistência à traça-do-tomateiro. Pesquisa Agropecuária Brasileira, v.34, p.733-740, 1999.

HADDAD, M.L.; MORAES, R.C.B.; PARRA, J.R.P. Sistema computadorizado para modelos estatísticos aplicados à entomologia. In: CONGRESSO BRASILEIRO DE ENTOMOLOGIA, 15., Caxambu, 1995. Resumos. Caxambu: SEB, 1995. p.244.

HAJI, F.N.P.; PARRA, J.R.P.; SILVA, J.P.; BATISTA, J.G.S. Biologia da traçado-tomateiro sob condições de laboratório. Pesquisa Agropecuária Brasileira, v.23, p.107-110, 1988.

JUVIK, J.A.; STEVENS, M.A. Physiological mechanisms of host-plant resistance in the genus Lycopersicon to Heliothis zea and Spodoptera exigua, two insect pests of the cultivated tomato. Journal of the American Society for Horticultural Science, v.106, n.6, p.1065-1069, 1982.

KENNEDY, G.G; SORENSON, C.F. Role of glandular trichomes in the resistance of Lycopersicon hirsutum f. glabratum to colorado potato beetle (Coleoptera: Chrysomelidae). Journal of Economic Entomology, v.78, p.547-551, 1985.

LEITE, G.L.D.; PICANÇO, M.; DELLA LUCIA, T.M.C.; MOREIRA, M.D. Role of canopy height in the resistance of Lycopersicon hirsutum f. glabratum to Tuta absoluta (Lep., Gelechiidae). Journal of Applied Entomology, v.123, p.459-463, 1999.

LOURENÇÃ̃, A.L.; NAGAI, H.; SIQUEIRA, W.J.; FONSECA, M.I.S. Seleção de linhagens de tomateiro resistentes a Scrobipalpula absoluta (Meyrick). Horticultura Brasileira, v.3, p.77, 1985.

MALUF, W.R.; BARBOSA, L.V.; SANTA CECÍLIA, L.V.C. 2-tridecanonemediated of mechanisms of resistance to the South American tomato pinworm Scrobipalpuloides absoluta (Meyrick, 1917) (Lepidoptera Gelechiidae) in Lycopersicon spp. Euphytica, v.93, p.189-194, 1997.

MIHSFELDT, L.H. Biologia e exigências térmicas de Tuta absoluta (Meyrick, 1917) em dieta artificial. Piracicaba, 1998. 87p. Tese (Doutorado) - Escola Superior de Agricultura "Luiz de Queiroz", Universidade de São Paulo.

PARRA, R.P.P.; HADDAD, M.L. Determinação do número de ínstares de insetos. Piracicaba: FEALQ, 1989, 49p.

QUIROZ, C.E. Nuevos antecedentes sobre la biologia de la polilla del tomate, Scrobipalpula absoluta (Meyrick). Agricultura Técnica, v.36, p.82-86, 1976.

RAZURI, V.; VARGAS, E. Biologia y comportamiento de Scrobipalpula absoluta Meyrick (Lep., Gelechiidae) en tomatera. Revista Peruana de Entomologia, v.18, p.84-89, 1975.

REIS FILHO, W.; VENDRAMIM, J.D.; FERNANDES, L.C. Influência de clones de seringueira (Havea spp.) na biologia de Erinnyis ello ello (L., 1758) (Lepidoptera, Sphingidae). Anais da Sociedade Entomológica do Brasil, v.15, (suplemento), 93-103, 1986.

SLANSKY Jr., F.; RODRIGUEZ, J.G. Nutritional ecology of insects, mites, spiders, and related invertebrates: an overview. In: SLANSKY Jr., F.; RODRIGUEZ, J.G. (Ed.). Nutritional Ecology of Insects, Mites, Spiders, and Related Invertebrates. New York: J. Wiley \& Sons, 1987. p. 1-69.

SOUZA, J.C. de; REIS, P.R. Traça-do-tomateiro, histórico, reconhecimento, biologia, prejuízos e controle. Belo Horizonte: EPAMIG, 1992. 19p. (EPAMIG. Boletim, 32).

VARGAS, C.H. Observaciones sobre la biología y enemigos naturales de la polilla del tomate, Gnorimoschema absoluta (Meyrick), (Lep. Gelechiidae). Idesia, v.1, p.75-110, 1970.

Recebido em 20.03.01 